\title{
AUTOETNOGRAFÍA, ESPACIO, IDENTIDAD Y RESISTENCIA EN LA NARRATIVA FUNDACIONAL DE GUINEA ECUATORIAL: CUANDO LOS COMBES LUCHABAN (1953) DE LEONCIO EVITA ENOY
}

\author{
POR \\ Rosario M. DE SwANSON \\ Marlboro College
}

In this great future, you can't forget your past...

Bob Marley

Publicada en Madrid en 1953 por el Instituto de Estudios Africanos (IDEA), Cuando los combes luchaban (Novela de costumbres de la Guinea Española) de Leoncio Evita Enoy se considera la primera novela guineoecuatoriana. Sin embargo, a pesar de su reconocimiento como texto fundacional de las letras guineanas, la novela ha provocado cierto malestar, cuando no un debate, tanto entre escritores como críticos guineanos que la han colocado bajo la rúbrica de literatura de consentimiento (Ngom, Osubita) ${ }^{1} \mathrm{o}$ la consideran literatura colonial (Bibang Oyee, Ilombé, Bokesa). ${ }^{2}$ De cierto modo, su recepción crítica ilustra las tensiones que acosan a las literaturas surgidas en medio de circunstancias coloniales en el mundo hispano.

El propósito del presente ensayo es doble. Primero, se propone examinar el texto de Evita Enoy como paradigma de lo que Mary Louise Pratt llama texto autoetnográfico que, junto con otros textos surgidos en un espacio colonial, despliega una perspectiva local que revela el espacio interior del texto como sitio de saberes impugnados, de conocimientos en disputa. Segundo, interesa ver cómo dentro de la perspectiva local, el conocimiento del espacio y la identidad, anclados en el discurso etnológico, se conjugan hasta llegar a formar un discurso de resistencia que responde a la mirada europea desde el interior del texto mismo. A través de ella se desestabilizan e interrogan formas dominantes de organización del conocimiento, es decir, la historia. Hecho importante si se considera que la construcción de la historia como forma superior de organización del

\footnotetext{
1 Sus comentarios están recogidos en los primeros artículos seminales que intentan esclarecer el lugar de las letras guineoecuatorianas en el ámbito hispano: "La literatura africana de expresión castellana" de Mbare'Ngom (412) y "Una introducción a la literatura guineana" de Juan Bautista Osubita en Quimera (46).

2 Mbare'Ngom recoge las opiniones de estos escritores en Diálogos con Guinea.
} 
pensamiento es inseparable de la reconstitución del espacio en la perspectiva occidental (Rabasa 356). El desarrollo de la cartografía moderna es paralelo al surgimiento de la historia (escrita) como discurso legitimador de la expansión territorial, económica y religiosa del occidente por la cual se intensificó la necesidad de organización y control espacial (Mignolo 40-41). La cartografía, como soporte visual y científico, legitima la colonización del espacio en el que Europa inscribe su historia (Rabasa 356). Cuando los combes luchaban, en tanto que despliega una perspectiva local anclada en el discurso etnológico, constituye el punto de inserción de la consciencia guineana en la occidental y como tal forma parte de una serie de desafíos a formas dominantes de organización del conocimiento desde la perspectiva de "los condenados de la tierra", como dijera Frantz Fanon. ${ }^{3}$ De ahí que la lucha de los guineanos se inscriba en un marco más amplio que comprende además, la lucha de los afrodescendientes en Latinoamérica y Europa.

La novela fue escrita hacia el ocaso del régimen colonial español en Guinea caracterizado por una censura y represión astringentes. En el prólogo a la primera edición, Carlos González Echegaray, censor y prologuista de la misma, la considera "una obrita francamente aceptable diferente de los relatos inconexos y absurdos que algunos 'morenos' seudointelectuales escriben" (11). Además, González Echegaray se maravilla de que, según su juicio, "la novela está pensada y sentida 'en blanco;' y sólo cuando la acción se desarrolla entre indígenas, solamente en parte, y como espectador, el escritor se siente de su raza" (12). Con todo, y pese a múltiples correcciones, la juzga ser un valioso "testimonio de costumbres y ritos hoy desaparecidos" escrito por un "indígena evolucionado" (12). La novela fue usada por el gobierno de Francisco Franco como muestra de los efectos positivos de la misión civilizadora de España en el África subsahariana (Ngom, "The Missing” 13). De ahí que, aunque reconocida como la primera novela guineoecuatoariana, Cuando los combes luchaban fuera puesta inicialmente bajo la rúbrica de literatura de consentimiento. Sin embargo, como advierte el escritor y crítico Donato Ndongo, tal valoración responde a factores externos y no a un análisis riguroso de la obra ("Literatura moderna" 126). ${ }^{4}$ Por su parte, Marvin Lewis aclara que aun cuando sea cierto que la novela está escrita desde la perspectiva de un sujeto colonizado, es erróneo asumir que carece de un discurso de resistencia y afirmación de valores africanos (111).

Un análisis más profundo revela que aunque es verdad que el texto integra modos discursivos europeos, lo hace para contrarrestarlos a través de una perspectiva

3 Según Mary Louise Pratt, la crítica del imperio ha sido continuamente expresada desde otros modos de expresión que incluyen "ceremony, dance, parody, philosophy, counterknowldge and counterhistory, in texts unwitnessed, supressed, lost, or simply overlain with repetition and unreality" (Imperial Eyes 2).

4 Para mayor discusión de Cuando los combes luchaban como literatura de consentimiento véase $A n$ Introduction to the Literature of Equatorial Guinea de Marvin Lewis (101-11) y la entrevista de Donato Ndongo en Diálogos con Guinea (69-90).

Revista Iberoamericana, Vol. LXXX, Núms. 248-249, Julio-Diciembre 2014, 777-789 
local que pone en entredicho la mirada europea. Por esta razón, Cuando los combes luchaban podría ser mejor entendido como un texto autoetnográfico producido en lo que Mary Louise Pratt llama la zona de contacto. Pratt define la zona de contacto como un espacio en el cual "cultures meet, clash, and grapple with each other, often in the context of highly asymmetrical relations of power, such as colonialism, slavery, or their aftermaths" ("Arts" 34). Mientras que en los textos etnográficos "European metropolitan subjects represent their others to themselves;" los textos autoetnográficos son "the representations that the so defined others construct in response to or in dialogue with those texts" ("Arts" 34). Los textos autoetnográficos constituyen el punto de entrada del grupo marginado en los circuitos de la cultura letrada dominante. Más aún, puesto que van dirigidos tanto a un lector metropolitano como a la comunidad del autor, se escriben en más de una lengua y suponen la cooperación de intelectuales y ancianos guardianes de la tradición oral ("Arts" 35). Perspectiva validada por Evita Enoy que, comentando acerca de las condiciones que predominaban en Guinea al momento de escribir, ha dicho: "La situación colonial que prevalecía cuando escribí mi novela, me dio un gran estímulo para seguir escribiendo y ampliar mis conocimientos. Personalmente, sentí gran satisfacción por abrir aquella pequeña brecha en el 'dique' del monopolio de la discriminación intelectual” (Ngom, Diálogos 33). Con estas palabras, Evita Enoy demuestra estar consciente del valor ético e intelectual de su obra: la novela atestigua la existencia de una tradición intelectual guineana que hasta entonces no había tenido una "voz" escritural. A través de ella se desmienten y desautorizan prácticas coloniales que habían marginado la tradición intelectual guineana al espacio del silencio.

A finales del siglo XIX, las Américas constituían, con algunas excepciones, un espacio emancipado, si bien fragmentado por la huella colonial lingüística y por la fortaleza o debilidad de sus recursos económicos. Aunque los lazos de España con África se remontan al siglo $\mathrm{XV}^{5} \mathrm{y}$ durante el siglo xIX se hizo hincapié en la colonización de Marruecos y el Sahara Occidental (Decalo 34-35), fue sólo después de 1898, cuando España había perdido sus últimas colonias americanas, que se gestó interés por desarrollar el cultivo de palma y cacao principalmente en la isla de Bioko (Fernando Poo) (Clarence-Smith 179). Como resultado de la guerra de 1898, los Estados Unidos surgieron como herederos de la empresa colonial europea en América, mientras que España, privada de sus colonias americanas, se volvió hacia África y Asia en un esfuerzo por mantener su poder (Elena y Ordóñez 78). A partir de entonces Guinea Ecuatorial y Marruecos tomarían el lugar de América dando lugar a una nueva reconfiguración del imaginario imperial dentro de España; cambio que produjo una intensificación de la presencia española en África, acicateada por la presencia francesa en la región que hoy comprende Gabón y Camerún (Sampedro 343).

Leslie Rout, The African Experience in Spanish America 1502 to the Present Day (5-17).

Revista Iberoamericana, Vol. LXXX, Núms. 248-249, Julio-Diciembre 2014, 777-789 
Es en esta coyuntura particular, que podemos ver cómo el espacio de Guinea comienza a ser codificado para dar cabida a una perspectiva europea; no obstante, también es posible discernir una perspectiva local que entra en contradicción con esta última. Existe en la estructura y lenguaje de la novela una resistencia tenaz a la mirada europea, una crítica disimulada, mas no oculta, de la colonización. La novela abre con un juego de luz y sombras: Brother John, un personaje blanco, despierta en medio de una noche muy oscura, "aquella noche era obscurísima" dice el narrador (37). Después de la reparación de los daños hechos por la pantera al dormitorio de las internas guineanas, Brother John entra en un profundo sueño justo al tiempo que la luz de la mañana llega a Ndyebengo y es recibida plenamente por Roku-a-Madiba, Rey de los combes (41). Así, desde el principio vemos que la figura del personaje blanco aparece rodeada de sombras mientras que la figura del Rey combe aparece rodeada de luz. Que la figura del Rey combe aparezca rodeada de luz no es sorprendente puesto que los combes derivan su nombre de la palabra "Ekombe" que quiere decir "los que arreglan las cosas", "los que van buscando el camino" y "alumbrado de los caminantes" (Iyanga Pendi 9).

Además, aunque Roku (también llamado Upolo), es descrito por el narrador como conforme con la perspectiva colonial, existen numerosos detalles enhebrados en la tesitura de la narración que contradicen esta postura. Por ejemplo, se nos dice que a pesar de que Roku "era salvaje” y "despreciaba las creencias del país. Su corazón firme le hacía desconfiar hasta de su propia sombra" (43). En el mismo párrafo una nota al calce nos informa que la población combe ha disminuido considerablemente desde la llegada de los europeos. ${ }^{6}$ Un poco más adelante Roku dice: "Los blancos [...] han sido enviados por Rambé (Dios) para que nos traigan la civilización”, pero el narrador nos aclara que "el salvaje no sabía atribuir a los blancos la precisa calificación, éstos a pesar de su interés por traficar, habían traído la más sana intención de civilizar" (43). Aquí, la yuxtaposición entre buenas intenciones y la trata de esclavos añade una dimensión aun más sombría al claroscuro invertido con el que da comienzo la novela. El juego de luz y sombras apunta simultáneamente a la Ilustración y a la base oscura de sus fundamentos. La imagen evoca lejanamente la misión civilizadora relatada en el informe sobre la "Supresión de las costumbres salvajes" de la novela El corazón de las tinieblas (113). ${ }^{7}$ Nos recuerda que la misión civilizadora estuvo también imbricada

6 Todas las citas de la novela provienen de la segunda edición realizada por Donato Ndongo en 1996 que incluye un segundo prólogo de González Echegaray, la presentación de Iyanga Pendi y un estudio introductorio a cargo del mismo Ndongo.

7 El título original de la obra es Heart of Darkness, publicada en 1899 en entregas periódicas en la revista inglesa Blackwood. La edición citada es de Jorge Luis Marzo, traducción de Sergio Pitol. Texto completo en http://soymenos.net/Conrad.pdf.

Revista Iberoamericana, Vol. LXXX, Núms. 248-249, Julio-Diciembre 2014, 777-789 
en la negación de la humanidad de ciertos seres humanos y en la erosión de formas de cultura valiosas.

Desde una óptica local se discierne también la misma dialéctica. El camino seguido por el Rey combe desde Ndyembengo hacia el lugar donde esconde el vino de palma se describe como "nada extraordinario, una línea que surcaba la tierra en varias direcciones: por los montes, por la espesura, por las praderas, por los pantanos. Algunas veces parecía cunetas en las que las aguas se deslizaban silenciosamente, y otras, se tornaba inaccesible" (42-43). La perspectiva asumida por el narrador percibe montañas, matorrales, praderas y pantanos, pero no un camino. Sabemos que hay un camino porque un hombre lo sigue. El camino desaparece tan pronto como el hombre se desplaza, sin dejar detrás suyo rastro alguno de la existencia de un camino. Desde afuera, sin la ayuda del conocimiento local, el espacio africano se torna incomprensible. Como señala Michel de Certeau, esto se debe a que el conocimiento del espacio "derives from the ensemble of movements deployed within it. Space occurs as the effect produced by the operations that orient it, situate it, temporalize it, and make it function [...] In short, space is a practiced place" (117).

La incomprensión del paisaje africano produce malestar y miedo, los cuales se traducen en la voluntad de dominar el espacio y sus habitantes. Brother John se refiere a África como "madre de todos los peligros" (50). Los españoles Martín Garrido y su sobrino Carlos, acompañados por el ex esclavo cubano Adonis (101), nos dicen, al final de la novela, que no podrán descansar "hasta que todas estas hermosas tierras estén bajo la soberanía de España" (124), revelando el carácter colonial de sus exploraciones. En la cita anterior, el espacio africano constituye el espacio de la otredad, el espacio de lo desconocido. Pero además, la cita ilustra los límites del conocimiento occidental, de ahí que el espacio africano sea visto, según sugiere Wlad Godzich, como "a threat to be reduced, a potential same-to-be, a yet-not same" (xiii). El modelo paradigmático en función, cuyo paralelo histórico sería la colonización del espacio y geografía hispanoamericanos, es el de la búsqueda, típica de los romances de caballería, en la que el caballero sale de su reino - el espacio de lo conocido- y se lanza a la conquista de la otredad. ${ }^{8}$ La búsqueda termina cuando el espacio desconocido es puesto bajo la esfera del sistema de valores hegemónicos imperantes en la corte del caballero (xiii). La exploración del territorio desconocido comprende la reinscripción del espacio africano adaptada a los modos de conocimiento europeos concretados en el mapa. El mapa, como herramienta matemática y geométrica, facilita la colonización ya que

8 América es el antecedente imperial de Guinea Ecuatorial como se observa repetidamente en la novela o como explica González Echegaray en su prólogo a la edición de 1996, citada en este trabajo: "Se pensaba oficialmente y se trataba de imbuir al indígena un pensamiento monolítico 'imperial' a base de evocaciones teóricas de las Leyes de Indias y de las grandezas pasadas de los españoles” (14).

Revista Iberoamericana, Vol. LXXX, Núms. 248-249, Julio-Diciembre 2014, 777-789 
rinde como vacío un espacio habitado. A través del mapa, el conocimiento local se sustituye por geometría abstracta, transformando el espacio en un sistema aislado de referencias geográficas desprovisto de presencia humana y de las prácticas culturales que lo produjeron como tal (de Certeau 121). Al eliminar a las personas que lo habitan, el entorno natural se presenta como un conjunto de recursos listos para su explotación.

Además, comprendidos en el significado del significante "tierras" se encuentran los guineanos. Esta estrategia permite no solamente la disposición de la tierra y de sus recursos naturales, sino también de las poblaciones que la habitan. Las diferentes etnias guineanas forman parte de los recursos listos para ser explotados, como se sugiere en la referencia a la trata de esclavos; o, como indica la presencia de Brother John, un grupo cuyos recursos serán intercambiados por la religión y civilización occidentales. Tal es el trueque económico revelado por la narrativa de la novela.

En el texto tres grupos: norteamericanos, franceses y españoles intentan ganar control sobre el espacio africano. Los norteamericanos Brother John y su esposa Leona pretenden educar y evangelizar a los combes; sin embargo, su verdadera finalidad es obtener el terreno de Bolondo, rico en recursos naturales (48). Por otra parte, el espectro del imperio, encarnado en la figura de Martín Garrido, "ex oficial de la Armada Española y ahora amante de África", y en la ambigua figura de Adonis, el ex esclavo cubano transformado en aventurero que acompaña su expedición a las costas de Guinea, está presente a lo largo de la narrativa (98). La novela marca el desplazamiento de los intereses coloniales de América a Guinea Ecuatorial. Según Adam Lifshey, la figura de Adonis, en tanto que comparte raíces africanas y es fiel a los españoles, reproduce la jerarquía social y opera como modelo a seguir para los llamados "indígenas" guineanos (124). La novela demuestra que los parámetros geográficos y temporales de la guerra entre Estados Unidos y España deben ser reexaminados para incluir la representación discursiva, desde África, de una realidad que era globalizada en ese momento y que ha sido globalizada desde entonces (Lifshey 132). Los acontecimientos narrados en la novela anticipan las luchas futuras que Guinea habría de librar en el presente. En cierto modo, la colonización es el trasfondo más amplio dentro del cual se enmarca la lucha por ser de los combe, invocada en el título de la novela.

En consonancia con el carácter dialógico de su obra, Evita Enoy enhebra la historia interior de los combes con la historia colonial de España en otros lugares. Esta doble perspectiva permite que tanto el lector guineano como el lector no guineano puedan situar los acontecimientos narrados en la novela en un contexto histórico determinado. El lector guineano sabrá que si bien algunos de los hechos narrados corresponden a la historia imaginada, no es así la lucha de los combes como tampoco Vilungua, el hijo del Rey. Para los combes, Vilungua va Macheba es un personaje histórico considerado como uno de los pensadores más iluminados de su tiempo. Su liderazgo frente a la penetración europea se destaca como uno de los más importantes de finales del siglo

Revista Iberoamericana, Vol. LXXX, Núms. 248-249, Julio-Diciembre 2014, 777-789 
XIX (Iyanga Pendi 19-20). Para el lector no guineano, en cambio, Martín Garrido y la ambigua figura de Adonis, en tanto que conjuran el espectro del imperio en conexión con España, introducen además una línea de tiempo exterior que nos permite situar los eventos narrados en la novela a finales del siglo XIX, después de la abolición de la esclavitud en Cuba (1886) y después de la independencia de Cuba y la anexión de Puerto Rico a los Estados Unidos (1898). ${ }^{9}$ Al hacer referencia al pasado histórico, Evita Enoy integra la historia local de Guinea a la historia humana universal (Ricoeur 52). Al mismo tiempo, la perspectiva bifocal inserta el pasado histórico y la lucha guineanas dentro de un continuum histórico más amplio, a través del cual, el autor vincula la lucha de los guineanos con la lucha de los afrodescendientes en Latinoamérica y Europa.

El tema de la resistencia enlazado a la identidad combe se introduce abiertamente mediante un discurso pronunciado por un orador anciano en nombre del Rey. Después de la desaparición de Vilungua (el hijo del Rey también llamado Vilangua) el suegro de Roku le reclama no haberlo educado en "las cosas nuevas que esos benditos hombres de otro color nos traen" como manera de protección (53). Como respuesta Roku convoca una asamblea general en la que un anciano pronuncia el siguiente discurso: "Hermanos: voy a aclarar un punto, con respecto a lo que nuestro suegro se refería a los blancos; antes de que Vilangua pasara en manos de esos hombres, necesitaba, como negro que era, conocer a fondo las cosas de su país natalicio, tales como cazar, pescar, etc., en una palabra vivir como negro genuino" (54). El mensaje del anciano es claro: antes de aprender de otras culturas, los combes no deben olvidar sus orígenes o abandonar sus prácticas culturales porque ello equivaldría a perder la memoria y en consecuencia perderse a sí mismos. El final de su discurso está marcado por la siguiente advertencia:

La civilización de que tanto apetecemos es como una antorcha; alumbra mucho, pero quema todo lo que encuentra a su paso. ¡Padres e hijos! Tened cuidado de apartar la luz a vuestros jóvenes, porque mañana será para ellos su más grande remordimiento... esta misma "civilización" nos presentará muchas cosas que nos harán olvidar hasta de nuestro viejo forjador, como ha ocurrido en otras partes del mundo. Aparecerá la moneda, el pequeño disco de hierro que ellos dan valor; dominará nuestras voluntades; habéis de estar sirviendo a hombres cual mujeres. Tenéis el ejemplo de los senegaleses. ¿Es esta la vida que queréis dejar a vuestros hijos? ¡No! Dejad que los futuros hombres crezcan como las especies que la selva guarda... Hermanos: consideren esta última advertencia: nuestra educación, que los blancos llaman salvajismo, obedece a nuestra naturaleza. No podemos trocar nuestras costumbres, ni podemos renunciarlas tampoco, por la sencilla razón de que todo intento de borrar nuestro origen redundará en perjuicio de la raza. (54-55)

9 Véase Skidmore y Smith, Modern Latin America.

Revista Iberoamericana, Vol. LXXX, Núms. 248-249, Julio-Diciembre 2014, 777-789 
En principio, el discurso del anciano alude a una problemática similar que forma parte de la formación discursiva de la nación hispanoamericana: la lucha entre la civilización y la barbarie. ${ }^{10}$ Problemática que en Hispanoamérica surge muy temprano y es el tema central de la novela de la tierra y de las diferentes vertientes de la literatura indigenista. ${ }^{11}$ No obstante, como subraya Lifshey, por su defensa de valores y tradiciones africanas, el discurso recuerda el ensayo seminal "Nuestra América" de José Martí, pronunciado alrededor del mismo eje de tiempo cubierto en la novela (130).

Sin embargo, más importante aún es el hecho de que por medio del discurso sabemos que el orador está plenamente consciente de los efectos de la llamada civilización que, no sin ironía, Evita Enoy ha puesto entre comillas. En otros momentos esta ironía se manifiesta en la forma de hablar de la civilización occidental conocida como "la enfermedad" que ya afecta a Gabón (123) y domina a Senegal (55). Con respecto a Gabón, James Gardiner apunta que fue precisamente entre 1898 y 1899 que se cometieron los peores abusos e injusticias como consecuencia del régimen de concesión instalado en todo el Congo francés del cual Gabón formaba parte en aquella época (12). En lo referente a Senegal, 1886 marca la muerte de Lat Dior Diop, el héroe de la resistencia contra la penetración francesa; simbólicamente su muerte marca la culminación de la colonización francesa del territorio senegalés sacudido por el tráfico de esclavos y por constantes rebeliones (Ross 19-21). En efecto, este es uno de los momentos en los que el texto lanza una crítica directa al colonialismo y en los que el autor se solidariza con otros países de África que han sido sometidos al yugo colonial. La contienda verbal sustentada por los oradores demuestra el grado de discusión sostenido por los combes acerca del rol que la cultura occidental debe jugar dentro de su propio contexto cultural. ${ }^{12}$

Como contrapartida a la deshumanización y pérdida de valores, el autor yuxtapone, a lo largo de la narración, descripciones detalladas que forman tableaux vivant de las prácticas culturales combe de finales del siglo XIX. Estas prácticas incluyen la disposición de los pueblos combe en el territorio que hoy es Guinea, los aspectos comunitarios de su mando, la invocación de los ancestros antes de tomar decisiones importantes, la proclamación de sus hazañas durante la batalla y los bailes asociados con las prácticas del Bweti. Su mención cuidadosamente detallada y reiterada una y otra vez dentro de la narrativa está encaminada a rescatarlas del olvido, a fijarlas en la memoria del

${ }_{10}$ Véase Facundo: Civilización y Barbarie de Domingo Faustino Sarmiento.

11 Véase Foundational Fictions de Doris Sommer y "The Literature of Indigenismo" de René Prieto (138$63)$.

12 La contienda verbal como estrategia literaria y arma de resistencia aparece también, aunque en un contexto poscolonial, en Las tinieblas de tu memoria negra de Donato Ndongo. El sentimiento de malestar con respecto a la presencia francesa aparece también en la novela Ekomo de María Nsué. Nnanga y Ekomo se aventuran en territorio Fang en busca de una cura para Ekomo. Pero dado que esta porción del territorio Fang está ahora bajo el gobierno francés, sus leyes caen pesadamente sobre la viuda Nnanga que es tratada como una extraña (195-248).

Revista Iberoamericana, Vol. LXXX, Núms. 248-249, Julio-Diciembre 2014, 777-789 
presente. Mbare'Ngom argumenta que en estas obras la inserción del discurso etnológico (descripción repetitiva de rituales, costumbres, tradiciones y leyendas) tiene como objetivo principal contrarrestar el discurso colonial español a través de la generación alternativa de objetos de conocimiento acerca de los autores mismos, de su historia y de su cultura ("The Missing" 12). Por ello, como sugiere Dosinda García-Alvite, su acto de recuperación constituye en sí mismo un acto de resistencia (11).

Sin embargo y más importante aún, es el hecho de que Evita Enoy presenta a los combes como participantes conscientes y como constructores activos de su momento histórico. Lejos de ser un grupo carente de conocimiento, desprovisto de capacidad o de agencia, los combes saben que tanto Senegal y Gabón ya han sucumbido a la colonización. De hecho, la tensión provocada por la presencia europea es una de las causas principales de la división suscitada entre los combes. Así, un grupo disidente comandado por Penda Lengui, el chamán combe, intenta socavar el poder de Rokua-Madiba utilizando el culto Bweti. Mbomio Bacheng explica que entre los Fang y los Ndowé existe un conjunto de prácticas religiosas complementarias: el Bweti y el Mimbili (74). En el Bweti, los vivos se comunican con los espíritus de los muertos a través del consumo del "iboga", una planta alucinógena; en el Mimbili, el espíritu de una persona fallecida entra en el cuerpo de una persona viva. Además, Bacheng explica que entre los Fang, los traficantes de esclavos se conocían comúnmente como “el hombre leopardo cazador" (74). Según James Fernández, el resurgimiento del Bweti en el siglo XIX, en la región de Guinea Ecuatorial, constituye una respuesta intelectual a la evangelización ("The Affirmation" 441). Uno de los objetivos principales del Bweti fue "the re-establishment of contact with the ancestors too much abandoned under the influence of Christian evangelization" (Fernández, Fang Architectonics 28).

En la novela el culto Bweti tiene como objetivos principales expulsar al grupo europeo asentado en la región y amedrentar a sus simpatizantes, en este caso el Rey de los combes. La novela abre cuando la secta del Leopardo ataca los dormitorios de las internas guineanas en el asentamiento de Brother John. Después de este primer ataque la secta del Leopardo dirige sus esfuerzos a debilitar el mando del Rey y privarlo de un heredero al trono. Primero atacan la fuente de sustento: pollos, cabras y otros animales amanecen sacrificados o simplemente desaparecen; luego después de que muchos guerreros son sacrificados, secuestran a Vilungua $(58,80)$. De cierto modo, la secta del Bweti trata de controlar el poder de los hombres leopardo (los supuestos traficantes de esclavos) asumiendo el mismo avatar para combatirlos. Bajo el embozo de la máscara del leopardo, la secta del Bweti articula oposición a la interferencia europea. Así, paradójicamente, como dice Octavio Paz, la máscara, al ocultar revela un dolor que no puede ser divulgado de otra manera (26). La respuesta violenta articulada por la secta del Bweti forma lo que la crítica casi nunca menciona: la resistencia anticolonial rezagada en la historia perdida de los vencidos, rescatada en el texto por Evita Enoy.

Revista Iberoamericana, Vol. LXXX, Núms. 248-249, Julio-Diciembre 2014, 777-789 
En cierto sentido, el autor nos permite vislumbrar el contexto político en el que se suscita la influencia europea en la región pero también las raíces de la resistencia. Siguiendo la tradición, Roku resuelve entablar una guerra contra la secta del Leopardo, sin embargo, cuando Roku es casi asesinado y Ndyembengo se encuentra al borde del colapso, Vilungua, el verdadero héroe de la novela, regresa. Vilungua, representa por tanto una entidad mayor: la lucha por ser de los combes ante la inminente y dolorosa realidad colonial. Como nos recuerda Lifshey, como verdadero héroe y heredero del mando, Vilungua es la pieza central del discurso del anciano; es por Vilungua (la comunidad combe) que se defienden los valores y tradiciones combe. Es a través de Vilungua como sujeto acosado por la colonización que se emite "from the most marginal of colonized spaces [...] a sweeping indictment of a planetary struggle between rising and falling world powers" (130-31). Es con los conocimientos proporcionados por Vilungua y bajo su liderazgo que el contingente conformado por guerreros combes y aventureros españoles, que la secta del Leopardo es finalmente derrotada. En ese momento, para el Rey combe, un desafío desde el seno de su comunidad parece ser una amenaza mayor. Roku-a-Madiba sabe que con la muerte de su hijo, será Penda Lengui, el chamán combe líder de la secta Bweti, quien heredará el mando (73). Ante esta disyuntiva, decide aliarse con los españoles, que a diferencia de los franceses que ya han dominado Senegal y Gabón, aún no representan una amenaza (85-86). La novela termina con la derrota de la secta Bweti y el restablecimiento del poder del Rey a través de una alianza temporal con los españoles y el misionero americano. A cambio de su ayuda Roku-a-Madiba permite que los españoles extraigan "caucho en bruto, Almendra, aceite de palma y marfil" (104); Brother John es recompensado con el terreno de Bolondo.

Al final, la paz ha regresado a Ndyebengo y Roku-a-Madiba ha asegurado la supervivencia de su estirpe. No obstante, sabemos que esta paz es temporal. El escritor se asegura de que el lector sepa que las condiciones de vida de los combes han sido alteradas para siempre ya que los europeos entran en acuerdo propio: "Una carcajada amistosa selló el pacto entre los españoles y el americano en nombre de Upolo. Y allí mismo empezó la aventura que nunca fue superada en aquellos primeros días del resurgir del Continente negro" (100). El pacto entre el pastor americano y los exploradores españoles señala el comienzo de una influencia europea mayor en la zona (que se sugiere será heredada por la jefatura de Vilungua). La referencia a África como continente negro apunta, por un lado, a la futura exploración y explotación económica de sus tierras y recursos; y por otro, a la conquista espiritual de su pueblo y de sus formas culturales, representados respectivamente, por los exploradores españoles y por el pastor. La novela termina, pero el lector puede anticipar que las luchas de Guinea apenas han comenzado. Con el final de la novela un capítulo temprano en la historia interior de Guinea Ecuatorial llega a su fin y da comienzo a lo que bien podríamos

Revista Iberoamericana, Vol. LXXX, Núms. 248-249, Julio-Diciembre 2014, 777-789 
llamar la historia moderna. Lo que llama la atención aquí es el hecho de que, al tiempo que América Latina se convierte en un espacio emancipado y neocolonial (en el caso de Puerto Rico), entrando en la carrera hacia la modernidad, el espectro del colonialismo se alza sobre Guinea Ecuatorial y sobre África.

Como texto autoetnográfico, el espacio de la novela se convierte en un campo de batalla textual en el que se recrea la lucha por ser de los combes en medio de circunstancias históricas complejas aunque desde una perspectiva guineana. Si bien la novela utiliza modos discursivos europeos, también es verdad que esta mirada europeizante se ve constantemente asediada por el discurso del otro que, desde una perspectiva autóctona, toma la palabra para darnos una visión de su filosofía y praxis oral, y de su contexto sociocultural y político, a través del cual la somete a un interrogatorio constante. El final de la novela apunta simultáneamente en dos direcciones. Hacia las luchas interiores, libradas entre los mismos combes, conservadas en la memoria oral. Hacia la historia colonial, y paradójicamente moderna, de Guinea conservada en la tradición escritural, de la que Cuando los combes luchaban forma parte, en la que la lucha por ser de los combes se mantiene viva. Lucha representativa de todos los guineanos. De esta manera, al esclarecer el pasado histórico desde una perspectiva guineana, Evita Enoy ha efectuado lo que el filósofo italiano Gianni Vattimo considera "the appropriation of the meaning of history by those who actually make it" (28). La novela de Evita Enoy regresa al pasado legendario, justo al momento histórico en el que se suscita la dominación española para revelar las raíces de la resistencia guineana; y revelar a la vez, las líneas principales del conflicto que han determinado el presente de Guinea Ecuatorial. Con ello Evita Enoy demuestra que sólo reexaminando el pasado podemos avanzar hacia el futuro; que es mediante el conocimiento de nuestra historia y tradiciones que se puede intentar una comprensión más profunda de la problemática que dio origen a nuestro presente.

\section{BiBLIOGRAFÍA}

Certeau, Michel de. The Practice of Everyday Life. Berkeley: U of California P, 1984. Clarence-Smith, W.G. "African and European Cocoa Producers on Fernando Poo, 1880s to 1910s." The Journal of African History (1994): 179-99.

Conrad, Joseph. El corazón de las tinieblas. Jorge Luis Marzo, ed. Sergio Pitol, trad. $<$ http://soymenos.net/Conrad.pdf.>. 15 dic. 2009.

Decalo, Samuel. Psychoses of Power. African Personal Dictatorships. Boulder: Westview, 1989.

Elena, Alberto y Javier Ordóñez. "Science, Technology, and the Spanish Colonial Experience in the Nineteenth Century." Osiris $2^{\text {nd }}$ ser. 15 (2000): 70-82.

Evita Enoy, Leoncio. Cuando los combes luchaban. Donato Ndongo Bidyogo, ed. Madrid: Agencia Española de Cooperación Internacional, 1996.

Revista Iberoamericana, Vol. LXXX, Núms. 248-249, Julio-Diciembre 2014, 777-789 
Fernández, J.W. "The Affirmation of Things Past: Alar Ayong and Bwiti as Movements of Protest in Central and Northern Gabon."Protest and Power in Black Africa. Robert I. Rotberg y Ali A. Mazrui, eds. Nueva York: Oxford UP, 1970. 457-27.

Fang Architectonics. Philadelphia, PA: ISHI (Institute for the Study of Human Issues), 1977.

Fanon, Frantz. Los condenados de la tierra. México: Fondo de Cultura Económica, 1965. García-Alvite, Dosinda. "Recuperación de prácticas tradicionales de Guinea Ecuatorial en Cuando los combés luchaban de Leoncio Evita". Espéculo. Revista de Estudios Literarios (2008). <http://www.ucm.es/info/especulo/numero39/ccombes.html>. 15 mayo 2009.

Gardiner, David E. Historical Dictionary of Gabon. Metuchen, NJ: The Scarecrow, 1981. Godzich, Wlad. Prólogo. "The Further Possibility of Knowledge.” Michael de Certeau. Heterologies. Discourse on the Other. Brian Massumi, trad. Minneapolis: U of Minnesota P, 1986. viii-xxi.

González Echegaray, Carlos. "Prólogo a la 1ª Edición de 1953". Cuando los combes luchaban. Novela de costumbres en la antigua Guinea Española. Madrid: Agencia Española de Cooperación Internacional, 1996. 11-12.

Iyanga Pendi, Augusto. "Breve presentación”. Cuando los combes luchaban. Madrid: Agencia Española de Cooperación Internacional, 1996. 19-22.

Lewis, Marvin. An Introduction to the Literature of Equatorial Guinea. Columbia: U of Missouri P, 2007.

Lifshey, Adam. “"No podemos soñar': A Hispanophone African Literary Displacement of the Spanish American War of 1898." Hispanic Journal 27 (2006): 119-34.

Mbomio Bacheng, Joaquín. "Leoncio Evita o Cuando los combes luchaban, una obra trascedental". Afro-Hispanic Review (2000): 72-78.

Mignolo, Walter. "The Movable Center: Geographical Discourse and Territoriality During the Expansion of the Spanish Empire." Coded Encounters. Writing, Gender and Ethnicity in Colonial Latin America. Javier Cevallos-Candau y otros, eds. Amherst: U of Massachusetts P, 1994. 15-45.

Ndongo-Bidyogo, Donato. "Literatura moderna hispanófona en Guinea Ecuatorial". Jornada de Estudios africanos. Marta Sofía Pérez, ed. León: Universidad de León, 2001. 125-34. Las Tinieblas de tu memoria negra. Madrid: Editorial Fundamentos, 1987.

Ngom, Mbare' ."Entrevista a Leoncio Evita”. Diálogos con Guinea. Madrid: Ediciones Labrys 54, 1996. 31-36.

"La literatura africana de expresión castellana. La creación literaria en Guinea Ecuatorial." Hispania (1993): 410-18.

"The Missing Link: African Hispanism at the Dawn of the Millenium.”Arachne@ Rutgers: Journal of Iberian and Latin American Literary and Cultural Studies 1/1 (2002). <http://arachne.rutgers.edu/vol1_1contents.htm>. 15 mayo 2009.

Revista Iberoamericana, Vol. LXXX, Núms. 248-249, Julio-Diciembre 2014, 777-789 
Nsué, María. Ekomo. Madrid: Casa de África, 2007.

Osubita, Juan Bautista. "Se habla español. Una introducción a la literatura guineana". Quimera 112 (1992): 44-49.

Paz, Octavio. "Máscaras mexicanas". El laberinto de la soledad. México: Fondo de Cultura Económica, 1959. 26-41.

Pratt, Mary Louise. "Arts of the Contact Zone." Profession (1991): 33-40. Imperial Eyes. Travel Writing and Transculturation. New York: Routledge, 1992.

Prieto, René. “The Literature of Indigenismo." The Cambridge History of Latin American Literature. 3 vols. Roberto González Echevarría y Enrique Pupo-Walker, eds. Cambridge: Cambridge UP, 2001. 138-63.

Rabasa, José. "Allegories of Atlas." The Post-colonial Studies Reader. Bill Ashcroft, Gareth Griffiths, Helen Tiffin, eds. Londres: Routledge, 1995. 358-64.

Ricoeur, Paul. Time and Narrative. Vols. 1-3. Kathleen Blamey y David Pellauer, trads. Chicago: U of Chicago P, 1984.

Ross, Eric S. Culture and Customs of Senegal. Wesport, CT: Greenwood, 2008.

Rout, Leslie B. The African Experience in Spanish America 1502 to the Present Day. Nueva York: Cambridge UP, 1976.

Sampedro Vizcaya, Benita. "Rethinking the Archive and the Colonial Library: Equatorial Guinea." Journal of Spanish Cultural Studies 9 (2008): 341-63.

Sarmiento, Domingo Faustino. Facundo, civilización y barbarie. México: Porrúa, 1966. Skidmore, Thomas y Peter H. Smith. Modern Latin America. Oxford: Oxford UP, 1992.

Sommer, Doris. Foundational Fictions: The National Romances of Latin America. Berkeley: U of California P, 1991.

Sundiata, Ibrahim K. "State Formation and Trade: The Rise and Fall of the Bubi Polity, c.1840-1910." The International Journal of African Historical Studies. 27 (1994): 505-23.

Vattimo, Gianni. The End of Modernity: Nihilism and Hermeneutics in a Postmodern Culture. Cambridge: Polity, 1988. 
